Article

\title{
Zunyimycins $B$ and $C$, New Chloroanthrabenzoxocinones Antibiotics against Methicillin-Resistant Staphylococcus aureus and Enterococci from Streptomyces sp. FJS31-2
}

\author{
Yuhong Lü ${ }^{1, \dagger}$, Meiyun Shao ${ }^{1,+}$, Yinyin Wang ${ }^{1,+}$, Shengyan Qian ${ }^{1,+}$, Miao Wang ${ }^{1}$, \\ Yingquan Wang ${ }^{1}$, Xiaoqian Li ${ }^{1}$, Yuxin Bao ${ }^{1}$, Chengmin Deng ${ }^{2}$, Changwu Yue ${ }^{2, *}$, \\ Daishun Liu ${ }^{2, *}$, Ning Liu ${ }^{3}$, Minghao Liu ${ }^{3}$, Ying Huang ${ }^{3, *}$, Zehui Chen ${ }^{4}$ and Yonglin $\mathrm{Hu}^{4}$ \\ 1 Guizhou Key Laboratory of Microbial Resources \& Drug Development, Zunyi Medical University, \\ Zunyi 563003, Guizhou, China; 18585737637@163.com (Y.L.); meiyun216@126.com (M.S.); \\ yinyinwang0530@126.com (Y.W.); qianshengyan@126.com (S.Q.); wangmiao_good@163.com (M.W.); \\ yingquanwang@126.com (Y.W.); xiaoqianli0908@163.com (X.L.); baoyuxin8680915@126.com (Y.B.) \\ 2 Zunyi Key Laboratory of Genetic Diagnosis \& Targeted Drug Therapy, The First People's Hospital of Zunyi, \\ Zunyi 563003, Guizhou, China; dengcmin@126.com \\ 3 State Key Laboratory of Microbial Resources, Institute of Microbiology, Chinese Academy of Sciences, \\ Beijing 100101, China; fussliu@126.com (N.L.); lysf1987313@163.com (M.L.) \\ 4 Department of Clinical Laboratory Medicine, Zunyi Medical University, Zunyi 563003, Guizhou, China; \\ czhtyb@163.com (Z.C.); huyonglin5559@163.com (Y.H.) \\ * Correspondence: changwuyue@hotmail.com (C.Y.); ldsdoc@126.com (D.L.); huangy@im.ac.cn (Y.H.); \\ Tel.: +86-851-2860-9561 (C.Y.); Fax: +86-851-2860-9511 (C.Y.); \\ Tel./Fax: +86-851-2892-3030 (D.L.); +86-10-6480-7311 (Y.H.) \\ + These authors contributed equally to this work.
}

Academic Editor: Diego Muñoz-Torrero

Received: 9 January 2017; Accepted: 5 February 2017; Published: 8 February 2017

\begin{abstract}
This study performed an optimization of the fermentation conditions to activate the expression of the zunyimycin family biosynthesis genes of the zunyimycin-producing streptomycetes strain Streptomyces sp. FJS31-2. Bioassay-guided isolation and purification by varied chromatographic methods yielded two new compounds of the zunyimycin derivatives, namely, 31-2-7 and 31-2-8, accompanied with three known anthrabenzoxocinones family members of zunyimycin A, BE24566B, and chloroanthrabenzoxocinone. Their structures were elucidated by NMR, HRESIMS, IR, UV, and CD. Results showed that these two compounds were structurally similar to the previously reported compound zunyimycin A but differed in positions and number of chlorine atom substitution. The two novel compounds were called zunyimycins B and C. Antibacterial activity assay indicated that zunyimycin C showed a good inhibitory effect on the methicillin-resistant Staphylococcus aureus and Enterococci.
\end{abstract}

Keywords: zunyimycins; chloroanthrabenzoxocinones; antibacterial; activity; MRSA; Enterococci; streptomycetes

\section{Introduction}

Actinomycetes were known as the most important microorganisms for microbial-derived halogenated antibiotics because they produce numerous novel halogenated natural products with a variety of biological activities [1,2]. Currently, hundreds of actinomycete-derived halogenide with potent pharmacological activities have been reported [3], including the well-known nonribosomal peptide, vancomycin, which is extensively used to treat infections caused by methicillin-resistant 
Staphylococcus aureus [4,5]. However, antibiotics, including vancomycin, are also powerless when faced with the superbugs [6,7]. Accordingly, researchers have been desperately seeking new, effective drug options because of the potential for "no prescription" to occur [8]. Genome mining is an effective tool that is employed by an increasing number of scientists for novel natural product discovery from actinomycetes $[9,10]$. During the course of our investigation for novel halogenated natural products from streptomycetes [11], we isolated the chlorinated modified compounds of the type II polyketide BE24566B (1) and its chloroderivatives called zunyimycin A (2) from Streptomyces sp. FJS31-2 [12,13]. The current study reports the isolation and characterization of two new halogenated type II polyketides zunyimycin B (3) and zunyimycin C (4) accompanied by their three derivatives of zunyimycin A, BE24566B, and chloroanthrabenzoxocinone from the fermentation solid culture of Streptomyces sp. FJS31-2 (Figure 1). Antibacterial activity assay indicated that zunyimycins B and C showed good inhibitory effects on the methicillin-resistant $S$. aureus and Enterococci.

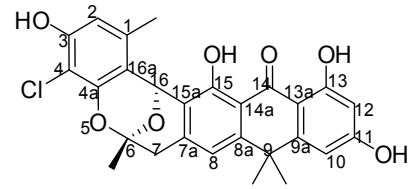

1

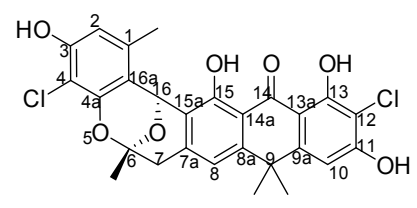

3

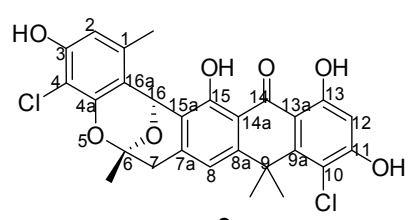

2

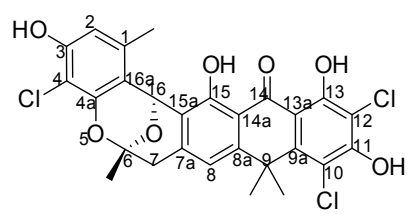

4

Figure 1. Chemical structure of zunyimycins from Streptomyces sp. FJS31-2.

\section{Results}

\subsection{Biosynthesis of Zunyimycins}

Among the 23 fermentation media, which were designed to screen the most effective medium for the biosynthesis of zunyimycins under the same conditions, Medium 22 was selected as the fermentation medium for zunyimycins because it exhibited the highest produced efficiency both on the product variety and the output among the four zunyimycin family numbers detected by HRESI-MS (Table 1). The results of the production testing of compounds by HPLC of the crude extracts from Streptomyces sp. FJS31-2 suggested that the four numbers of the zunyimycin family showed a different producing model under different incubation time (Figure 2). For example, the initiation of the biosynthesis of BE-24566B may be earlier than seven days and reach its peak on the 13th day. By contrast, zunyimycin A may start on the 9th day and reach its peak on the 13th day as well.

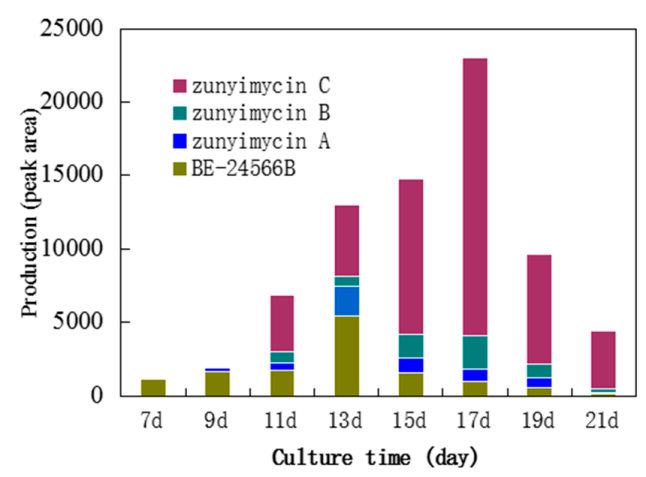

Figure 2. Biosynthesis model of the zunyimycin family. 
Table 1. Biosynthesis of zunyimycins.

\begin{tabular}{|c|c|c|c|c|}
\hline \multirow{2}{*}{ Medium } & \multicolumn{4}{|c|}{ Production (Peak Height, mAU) } \\
\hline & BE24566B & Zunyimycin A & Zunyimycin B & Zunyimycin C \\
\hline 1 & & & & \\
\hline 2 & 107 & & & \\
\hline 3 & & & & \\
\hline 4 & & & & \\
\hline 5 & 50 & & & \\
\hline 6 & 320 & & 135 & 190 \\
\hline 7 & & & & \\
\hline 8 & 124 & & & \\
\hline 9 & 122 & & & \\
\hline 10 & 200 & 52 & 24 & \\
\hline 11 & & & & 90 \\
\hline 12 & 75 & & & \\
\hline 13 & & & & \\
\hline 14 & 150 & & & \\
\hline 15 & 115 & & & \\
\hline 16 & 110 & & & \\
\hline 17 & & & & \\
\hline 18 & & & 100 & 108 \\
\hline 19 & 99 & & & \\
\hline 20 & 160 & & & \\
\hline 21 & & & & \\
\hline 22 & 525 & 250 & 526 & 824 \\
\hline 23 & 117 & & & 150 \\
\hline
\end{tabular}

\subsection{Chemical Identification of Zunyimycin B and $C$}

Zunyimycin B (3) was obtained a pale yellow powder and is soluble in dimethyl sulfoxide, $\mathrm{MeOH}$, and MeCOMe, among others. The IR spectrum of 1 showed absorption at $3385 \mathrm{~cm}^{-1}$ and $1609 \mathrm{~cm}^{-1}$ indicative of the presence of hydroxyl and carbonyl groups and the absorption at $1421 \mathrm{~cm}^{-1}$ and $1384 \mathrm{~cm}^{-1}$ illustrated the existence of benzene ring. HR-TOF-MS ion of the IR spectrum of $\mathbf{1}$ at $\mathrm{m} / \mathrm{z}$ $527.0658[\mathrm{M}]^{-}$indicated the molecular formula to be $\mathrm{C}_{27} \mathrm{H}_{22} \mathrm{Cl}_{2} \mathrm{O}_{7}$, thereby implying 16 degrees of unsaturation. The ${ }^{13} \mathrm{C}-\mathrm{NMR}, \mathrm{HSQC}$, and DEPT spectra of $\mathbf{1}$ displayed signals for 18 aromatic carbons at $\delta_{C} 160.6,157.7,152.6,151.8,150.7,150.7,150.4,141.2,133.4,122.5,117.2,115.7,113.6,111.2,107.2$, 106.3, and 101.7 which was similar to the ${ }^{13} \mathrm{C}-\mathrm{NMR}, \mathrm{HSQC}$, and DEPT spectra of BE-24566B $\left(\delta_{\mathrm{C}}\right.$ 167.7, 167.4, 159.6, 159.0, 157.0, 154.5, 152.4, 144.8, 138.0, 125.5, 119.5, 115.8, 113.2, 109.1, 108.4, 103.0, and 101.2) and zunyimycin A $\left(\delta_{C} 164.5,163.0,156.9,153.2,152.6,150.6,148.3,141.9,133.4,122.1,117.7\right.$, $115.4,112.6,109.9,107.6,102.6$, and 101.6) demonstrated that the constituent was anthrabenzoxocinone (ABX). In addition, combined with the signal of carbonyl group of $\delta_{C} 189.7$, the methyl group at $\delta_{C}$ 39.4, quaternary carbon at $\delta_{C} 38.1$, and carbon proton at $\delta_{C} 98.4$ indicated that Compound 3 had the skeleton of ABX. Moreover, the ${ }^{1} \mathrm{H}-\mathrm{NMR}$ spectra of 1 that display signals at $6.82(1 \mathrm{H}, \mathrm{s}), 6.57(1 \mathrm{H}, \mathrm{s})$, $6.17(1 \mathrm{H}, \mathrm{s}), 2.41(3 \mathrm{H}, \mathrm{s}), 1.51(3 \mathrm{H}, \mathrm{s}), 1.45(3 \mathrm{H}, \mathrm{s})$, and $1.36(3 \mathrm{H}, \mathrm{s})$ also demonstrate the presence of ABX. Table 2 shows the NMR data. The HMBC experiment showed correlation between the proton at $\delta_{\mathrm{H}} 6.82$ and carbons at $\delta_{\mathrm{C}} 111.2$ and 122.5 , thereby indicating that one hydroxyl atom connected with the C-8 position. The signal at $\delta_{\mathrm{H}} 6.57(1 \mathrm{H}, \mathrm{s})$ and carbons at $\delta_{\mathrm{C}} 106.3$, combined with the ROSEY spectra showed a correlation with $\mathrm{H}-10 / 9-\mathrm{CH}_{3}$, thereby implying that the signal at $\delta_{\mathrm{H}} 6.57$ connected with the benzene ring at the $\mathrm{C}-10$ position. The proton signal at $\delta_{\mathrm{H}} 6.17(1 \mathrm{H}, \mathrm{s})$ correlated with $\delta_{\mathrm{C}}$ $152.6,150.7$, and 133.4, thereby indicating that the signals to $\delta_{\mathrm{H}} 6.17$ connected with the benzene ring at the $\mathrm{C}-2$ position. The ${ }^{1} \mathrm{H}-{ }^{1} \mathrm{H}$ COSY showed that the correlation $\mathrm{H}-2 / 1-\mathrm{CH}_{3}$ can also demonstrate the $\delta_{\mathrm{H}} 6.17$ connected with the $\mathrm{C}-2$ position. In accordance with the preceding information, the relative structure of Compound 3 was substituted by chlorine atoms at the C-4 and C-12 positions of ABX. 
Table 2. NMR data of zunyimycin B and $\mathrm{C}$ in $500\left({ }^{1} \mathrm{H}\right)$ and $125\left({ }^{13} \mathrm{C}\right) \mathrm{MHz}\left(\mathrm{MeOH}-d_{6}, \delta \mathrm{ppm}\right)$.

\begin{tabular}{|c|c|c|c|c|}
\hline \multirow{2}{*}{ Position } & \multicolumn{2}{|c|}{${ }^{1} \mathrm{H}$} & \multicolumn{2}{|c|}{${ }^{13} \mathrm{C}$} \\
\hline & Zunyimycin B & Zunyimycin C & Zunyimycin B & Zunyimycin C \\
\hline 1 & & & 133.4 & 133.4 \\
\hline 2 & $6.17(1 \mathrm{H}, \mathrm{s})$ & $6.27(1 \mathrm{H}, \mathrm{s})$ & 101.7 & 101.7 \\
\hline 3 & & & 150.7 & 150.7 \\
\hline 4 & & & 113.6 & 113.6 \\
\hline $4 a$ & & & 152.6 & 152.6 \\
\hline 5 & & & & \\
\hline 6 & & & 98.4 & 98.3 \\
\hline 7 & $\begin{array}{l}3.04(1 \mathrm{H}, \mathrm{d}, J=11.3) \\
2.93(1 \mathrm{H}, \mathrm{d}, J=17.5)\end{array}$ & $3.04-3.11(2 \mathrm{H}, \mathrm{m})$ & 39.6 & 39.7 \\
\hline $7 \mathrm{a}$ & & & 141.2 & 142.4 \\
\hline 8 & $6.82(1 \mathrm{H}, \mathrm{s})$ & $6.86(1 \mathrm{H}, \mathrm{s})$ & 117.2 & 117.9 \\
\hline $8 a$ & & & 150.7 & 146.4 \\
\hline 9 & & & 38.1 & 39.4 \\
\hline $9 \mathrm{a}$ & & & 150.4 & 153.0 \\
\hline 10 & $6.57(1 \mathrm{H}, \mathrm{s})$ & & 107.2 & 107.9 \\
\hline 11 & & & 160.6 & 159.6 \\
\hline 12 & & & 106.3 & 107.8 \\
\hline 13 & & & 151.8 & 157.4 \\
\hline $13 a$ & & & 113.6 & 109.7 \\
\hline 14 & & & 189.7 & 190.8 \\
\hline $14 a$ & & & 111.2 & 112.3 \\
\hline 15 & & & 157.7 & 157.1 \\
\hline $15 a$ & & & 122.5 & 122.1 \\
\hline 16 & $6.10(1 \mathrm{H}, \mathrm{s})$ & $6.10(1 \mathrm{H}, \mathrm{s})$ & 65.8 & 65.7 \\
\hline $16 a$ & & & 115.7 & 115.6 \\
\hline $1-\mathrm{CH}_{3}$ & $2.52(3 \mathrm{H}, \mathrm{s})$ & $2.49(3 \mathrm{H}, \mathrm{s})$ & 15.6 & 15.8 \\
\hline $6-\mathrm{CH}_{3}$ & $1.51(3 \mathrm{H}, \mathrm{s})$ & $1.60(3 \mathrm{H}, \mathrm{s})$ & 26.2 & 26.2 \\
\hline $9-\mathrm{CH}_{3}$ & $1.36(3 \mathrm{H}, \mathrm{s})$ & $1.79(3 \mathrm{H}, \mathrm{s})$ & 33.0 & 27.5 \\
\hline $9-\mathrm{CH}_{3}$ & $1.45(3 \mathrm{H}, \mathrm{s})$ & $1.69(3 \mathrm{H}, \mathrm{s})$ & 32.6 & 27.8 \\
\hline
\end{tabular}

Zunyimycin $\mathrm{C}(4)$ was obtained as a pale yellow powder. Its molecular formula $\mathrm{C}_{27} \mathrm{H}_{21} \mathrm{Cl}_{3} \mathrm{O}_{7}$ established on the basis of the HR-TOF-MS ion at $m / z 527.0658[\mathrm{M}]^{-}$implied that it is one chlorine atom more than zunyimycin B. The analysis of ${ }^{1} \mathrm{H}-,{ }^{13} \mathrm{C}-\mathrm{NMR}$, DEPT, COSY, and HSQC spectra (Table 2) was similar with zunyimycin $B$, thereby confirming that zunyimycin $C$ had the presence of ABX. The HMBC experiment showed a correlation between the proton at $\delta_{\mathrm{H}} 6.86$ and carbons at $\delta_{C} 112.3$ and 122.1, thereby indicating that one hydroxyl atom is connected with the C-8 position. The proton signal at $\delta_{\mathrm{H}} 6.17(1 \mathrm{H}, \mathrm{s})$ correlated with $\delta_{\mathrm{C}} 152.6,150.7,133.4,115.7$, and 113.6, thereby indicating that the signals to $\delta_{\mathrm{H}} 6.17$ is connected with the benzene ring at the $\mathrm{C}-2$ position. The ${ }^{1} \mathrm{H}-{ }^{1} \mathrm{H}$ COSY spectra showed that the correlation $\mathrm{H}-2 / 1-\mathrm{CH} 3$ can also demonstrate the $\delta_{\mathrm{H}} 6.17$ connected with the $\mathrm{C}-2$ position. In accordance with the preceding information, the relative structure of Compound 2 was substituted by chlorine atoms at the C-4, C-10, and C-12 positions of ABX. Figure 3 shows the 2D NMR data.

The absolute configuration of zunyimycins $B$ and $C$ was confirmed through the $C D$ spectrum (Figure 4). The CD spectrum of zunyimycins $B$ and $C$ displayed an apparent positive cotton effect (CD) at $239 \mathrm{~nm}$ and at $283 \mathrm{~nm}$, thereby indicating $2 \mathrm{~S}$ configurations for the C-16 and C-6 positions in the two compounds. 


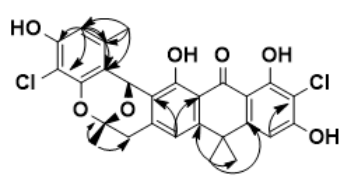

A HMBC $\overparen{\mathrm{HC}}$

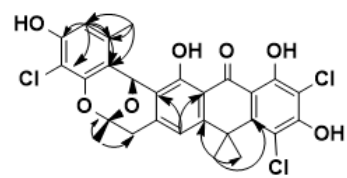

B
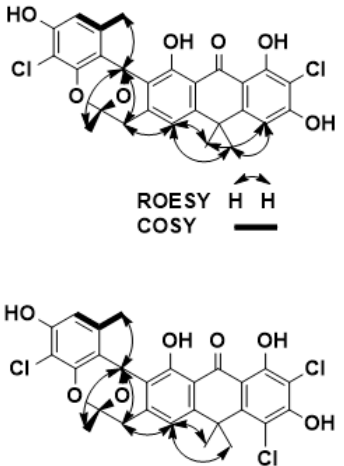

ROESY H H
COSY

Figure 3. HMBC and ROESY correlations for Zunyimycin B (upper, A) and C (lower, B).

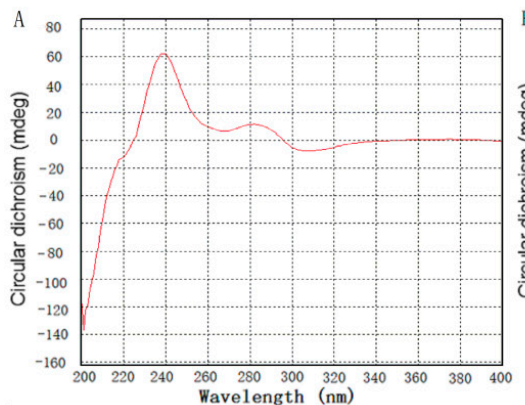

(a)

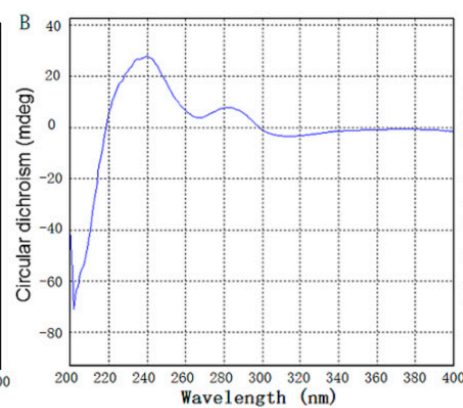

(b)

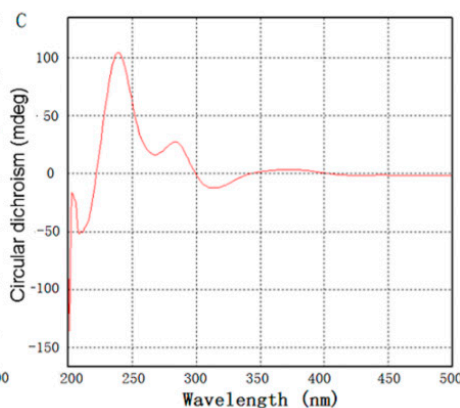

(c)

Figure 4. Circular dichroism spectrum of zunyimycin A (a); zunyimycin B (b) and zunyimycin C (c).

\subsection{Antibacterial Activity of Zunyimycins}

The results of the antibacterial activity testing showed that both zunyimycins A, B and C exhibited antibacterial activity against the Enterococci (Enterococcus faecalis), Bacillus (Bacillus subtilis) as well as both the methicillin-sensitive and methicillin-resistant Staphylococcus aureus. Among the three chloroanthrabenzoxocinones antibiotics, zunyimycin $C$ showing more higher antibacterial activity since the MICs against the Enterococci, Bacillus as well as both the methicillin-sensitive and methicillinresistant Staphylococcus aureus were lower than its analogues of zunyimycin A and B (Table 3).

Table 3. Minimum inhibitory concentrations of zunyimycins $(\mu \mathrm{g} / \mathrm{mL})$.

\begin{tabular}{ccccc}
\hline Strains & Ampicillin & Zunyimycin A & Zunyimycin B & Zunyimycin C \\
\hline S. aureus (ATCC: 29213) & 1.05 & 3.44 & 3.94 & 0.94 \\
MRSA clinical isolates (08301) & $>100$ & 6.89 & 7.88 & 3.75 \\
MRSA clinical isolates (161222330) & $>100$ & 16.71 & 25.62 & 8.14 \\
MRSA clinical isolates (161231380) & $>100$ & 8.36 & 12.81 & 4.07 \\
MRSA clinical isolates (170108317) & $>100$ & 16.71 & 25.62 & 4.07 \\
MRSA clinical isolates (161231350) & $>100$ & 16.71 & 25.62 & 4.07 \\
E. faecalis (ATCC: 29212) & $>100$ & 13.78 & 15.75 & 7.50 \\
E. faecalis clinical isolates (160803348) & $>100$ & 16.71 & 12.81 & 4.07 \\
E. faecalis clinical isolates (160804314) & $>100$ & 16.71 & 12.81 & 8.14 \\
E. faecalis clinical isolates (161222328) & $>100$ & 33.43 & 12.81 & 4.07 \\
E. faecalis clinical isolates (170106034) & $>100$ & 16.71 & 25.62 & 8.14 \\
B. subtilis (CGMCC: 1.2428) & 0.71 & 13.78 & 15.75 & 3.75 \\
\hline
\end{tabular}




\section{Discussion}

Currently, over 4500 halogenated natural products were isolated and generally with various biological activities, such as antibacterial, antitumor, and antiviral activities [14]. Halogenated natural products have been known as the most important resource of antibiotics because of their biological activity and their potential for medicinal use $[15,16]$. ABXs are a group of hexacyclics aromatic ketones with bioactivities [17], which were first isolated from Streptomyces violaceusniger. In the current study, two novel compounds, namely zunyimycins B and C, showed good inhibitory effect on the methicillin-resistant $S$. aureus, which were isolated from Streptomyces sp. FJS31-2 along with their analogues zunyimycin A and BE-24566B. In accordance with the results of the structure elucidation of zunyimycins A, B, and C, four modification sites for halogenation were discovered (Figure 5). The halogenation reactions of the three compounds may be conducted by $\mathrm{AbxH}$ because only one halogenase $(\mathrm{AbxH})$ was scanned in the genome of Streptomyces sp. FJS31-2. In spite of the catalytic mechanism that has not been precisely defined, $\mathrm{AbxH}$ may play an important role in the discovery of actinomycete-derived halogenated natural products. The halometabolites are not only highly diverse in biogenic origin, but also with respect to the halogen substitution patterns as well as chemical and structural complexity. Most halometabolites are biologically active, showing, e.g., antimicrobial, antifungal, or antibiotic activity. Since the biological activities of halometabolites are usually critically dependent on the presence (halogenated modification sites or number) of the halogen(s) [18], this may the reason of zunyimycin $C$ shows the best activities compared to its analogues.

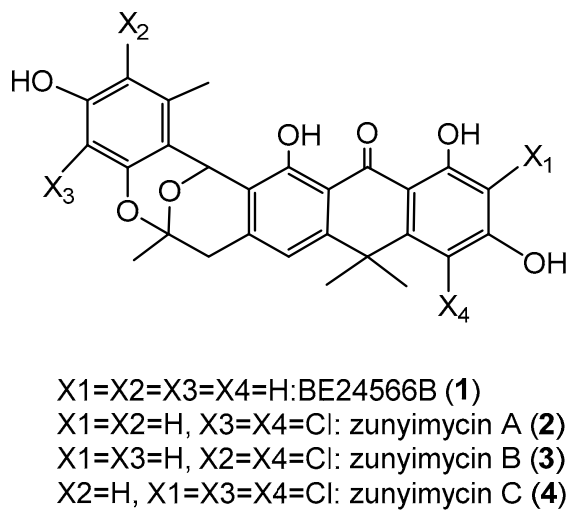

Figure 5. Proposed catalytic sites of $\mathrm{AbxH}$.

The results of the biosynthesis model analysis of the zunyimycin family suggest that under the same conditions, fermentation time may affect the output and compounds during fermentation. Hence, unlike BE-24566B and zunyimycin A, the biosynthesis of zunyimycins B and C started on the d11th day and reached its peak on the 17th day caused by the chloro-halogenation reaction of the compounds happening after the formation of the backbone of the compounds (Figure 2).

\section{Materials and Methods}

\subsection{Strains and Medium}

Five methicillin resistant Staphylococcus aureus clinical isolates and four Enterococcus faecalis clinical isolates were obtained from the Department of Laboratory Medicine of Zunyi Medical University. Staphylocccus aureus (ATCC: 29213), Enterococcus faecalis (ATCC: 29212) and Bacillus subtilis (CGMCC: 1.2428) were from China General Microbiological Culture Collection Center. Streptomyces sp. FJS31-2 was isolated from a soil sample collected from the Fanjing Mountain of Guizhou Province and was deposited in the China General Microbiological Culture Collection Center under accession number CGMCC 4.7321. The actinomycetic strain was preliminarily identified as a Streptomyces species based on morphological observation and physio-biochemical characteristics. The 16S rRNA 
gene was cloned by PCR and the DNA sequencing showing that it was substantially homologous (i.e., above $99 \%$ ) with the Streptomyces sparsogenes strain NBRC 13086 by multiple sequence alignment and phylogeny evolution analysis revealed that it was a variant species of $S$. sparsogenes. The genome DNA of Streptomyces sp. FJS31-2 was sequenced and deposited in GenBank under accession number PRJNA320463. The results of the antibacterial activity assay suggest that the strain exhibits biological activity against Candida albicans, B. subtilis, and M. luteus. The gene screening results of the secondary metabolism biosynthesis-associated genes showed that the coding region DNA sequence of halogenase, non-ribosomal peptide synthetases, type I polyketide synthase, and type II polyketide synthase (PKS II) were detected in the genome of Streptomyces sp. FJS31-2.

A total of 23 media with different carbon sources, nitrogen sources, and humic acid extract were designed to induce the biosynthesis of zunyimycins (Table 4). After culturing under the same conditions $\left(28^{\circ} \mathrm{C}\right.$, stationary culture) for $7,9,11,13,15,17,19$, and 21 days, the lawn plate including streptomycetes and culture medium were extracted with the same volume of ethyl acetate thrice. The production of the target compound from different media was detected through HPLC in accordance with the peak area of the ultraviolet absorption spectrum to optimize the high-producing culture condition of Streptomyces sp. FJS31-2 for zunyimycins.

Table 4. Medium for the biosynthesis of zunyimycins.

\begin{tabular}{|c|c|c|c|c|c|c|c|c|}
\hline \multicolumn{9}{|c|}{ Medium Components (g/L) } \\
\hline Medium & $\mathrm{CaCO}_{3}$ & Glucose & Malt Exact & Yeast Exact & Mannitol & $\mathrm{NH}_{4} \mathrm{NO}_{3}$ & Humic Acid A & Humic Acid B \\
\hline 1 & 2 & & 10 & & 4 & 4 & & \\
\hline 2 & 2 & & 10 & 4 & 4 & & & \\
\hline 3 & 2 & & 10 & 1 & 4 & & & \\
\hline 4 & 2 & & 10 & 2 & 4 & 2 & & \\
\hline 5 & 2 & 1 & 10 & & & 4 & & \\
\hline 6 & 2 & 1 & 10 & 4 & & & & \\
\hline 7 & 2 & 1 & 10 & 1 & & & & \\
\hline 8 & 2 & 1 & 10 & 2 & & 2 & & \\
\hline 9 & 2 & 4 & 10 & & & 4 & & \\
\hline 10 & 2 & 4 & 10 & 4 & & & & \\
\hline 11 & 2 & 4 & 10 & 1 & & & & \\
\hline 12 & 2 & 4 & 10 & 2 & & 2 & & \\
\hline 13 & 2 & 2 & 10 & & 2 & 4 & & \\
\hline 14 & 2 & 2 & 10 & 4 & 2 & & & \\
\hline 15 & 2 & 2 & 10 & 1 & 2 & & & \\
\hline 16 & 2 & 2 & 10 & 2 & 2 & 2 & & \\
\hline 17 & 2 & 1 & & 1 & & & & \\
\hline 18 & 2 & 4 & 10 & 4 & & & 0.5 & \\
\hline 19 & 2 & 4 & 10 & 4 & & & 1. & \\
\hline 20 & 2 & 4 & 10 & 4 & & & 1.5 & \\
\hline 21 & 2 & 4 & 10 & 4 & & & & 0.5 \\
\hline 22 & 2 & 4 & 10 & 4 & & & & 1.0 \\
\hline 23 & 2 & 4 & 10 & 4 & & & & 1.5 \\
\hline
\end{tabular}

Humic acid A: extract with water; Humic acid B: extract with alcohol. Each medium was supplemented with $1 \mathrm{~mL}$ trace elements $\left(\mathrm{ZnSO}_{47} \cdot \mathrm{H}_{2} \mathrm{O} 1 \mathrm{~g} / \mathrm{L}, \mathrm{FeSO}_{47} \cdot \mathrm{H}_{2} \mathrm{O} 1 \mathrm{~g} / \mathrm{L}, \mathrm{MnCl}_{2} \cdot 4 \mathrm{H}_{2} \mathrm{O} 1 \mathrm{~g} / \mathrm{L}, \cdot \mathrm{CuSO}_{4} \cdot 5 \mathrm{H}_{2} \mathrm{O} 1 \mathrm{~g} / \mathrm{L}, \mathrm{Na}_{2} \mathrm{~B}_{4} \mathrm{O}_{7} \cdot 10 \mathrm{H}_{2} \mathrm{O}\right.$ $\left.\left.1 \mathrm{~g} / \mathrm{L}, \mathrm{NH}_{4}\right)_{6} \mathrm{Mo}_{7} \mathrm{O}_{24} \cdot 4 \mathrm{H}_{2} \mathrm{O} 1 \mathrm{~g} / \mathrm{L}\right)$ in $100 \mathrm{~mL}$.

\subsection{Fermentation, Isolation, and Chemical Identification of BE-24566B and Zunyimycins $A, B$, and $C$}

In accordance with the results of the optimized culture conditions, Streptomyces sp. FJS31-2 was cultured using $140 \times 500 \mathrm{~mL}$ shake flasks containing $100 \mathrm{~mL}$ of ISP 2 agar medium with $10 \%$ natural humus acid water extracts. Thereafter, the culture was incubated for 13 days at $28^{\circ} \mathrm{C}$ to produce zunyimycin $\mathrm{B}$ and 17 days for zunyimycin $\mathrm{C}$. The solid culture was mashed and extracted thrice with $100 \mathrm{~mL}$ of ethanol in a shake flask at $28^{\circ} \mathrm{C}$ for $7 \mathrm{~h}$ at $110 \mathrm{rpm}$ after cultivation. Thereafter, the organic portion was concentrated in vacuo to remove the solvent. The crude extract was applied to silica gel column chromatography using the $\mathrm{CHCl}_{3} / \mathrm{MeOH}$ gradient to obtain the crude products. Further purification was conducted using Sephadex LH-20 (GE Healthcare, Tokyo, Japan) (MeOH) column 
and RP-HPLC (Shimadzu SPD-M20A with Xbridge ODS $10 \mathrm{~mm} \times 150 \mathrm{~mm}$ column). Compounds were identified using a HRESI-MS (Waters Xevo G2 QTOF mass spectrometer (Waters Corporation, Milford, MA, USA) and NMR (Bruker AV 600 MHz) (Bruker Corporation, Karlsruhe, Germany) for analysis [19].

\subsection{Antibacterial Activity Assay}

The antibacterial activity of zunyimycins A, B, and C was investigated against Staphylococcus aureus and Enterococci. Bacteria were grown at $37^{\circ} \mathrm{C}$ in Luria-Bertani (LB) broth (Difco, Sparks, MD, USA) with continuous shaking until the optical density $\left(\mathrm{OD}_{595}\right)$ reached 0.6. The bactericidal activity of zunyimycins A, B, and C was investigated using parameters such as the minimum inhibitory concentrations (MIC). The MIC values for zunyimycins $\mathrm{A}, \mathrm{B}$, and $\mathrm{C}$ were evaluated in quadruplet wells of sterile 96-well microtiter plates using the broth microdilution assay using the previously described broth microdilution procedure. Briefly, bacterial strains were clutured overnight at $35^{\circ} \mathrm{C}$ in liquid LB medium and test strains were suspended in fresh LB to yield a final density of $5 \times 10^{5}$ clolonies-forming units (cfu)/mL [20]. Geometric dilution ranging from 100 to $0.05 \mu \mathrm{g}$ of zunyimycins and DMSO solvent were prepared in a 96-well microtiter plate (50 $\mu \mathrm{L}$ of $\mathrm{LB}, 50 \mu \mathrm{L}$ of the zunyimycins and pure DMSO $+50 \mu \mathrm{L}$ of test strains). All plates were sealed lightly (with ventilation) and incubated thereafter at $35^{\circ} \mathrm{C}$ for $18 \mathrm{~h}$. The bacterial growth was indicated by the presence of white "pellet" on the well bottom. All tests were performed in quadruplets for test strains. The concentration of the first well with no turbidity was considered the MIC.

\section{Conclusions}

In summary, two novel compounds, namely zunyimycins $B$ and $C$ along with their analogous zunyimycin A and BE-24566B, were isolated from Streptomyces sp. FJS31-2. An antibacterial activity assay indicated that zunyimycins A, B and C showed antibiotic activity against methicillin-resistant S. aureus.

Acknowledgments: This work was funded by the National Nature Science Foundation of China (Grant No. 31160004, 31460006), the Program for Innovative Research Team in Guizhou Province (No. QKH-RCTD-20134035) and the Science and Technology Foundation of Guizhou Province (No. (2010)2156), (2012)2348), (2013)3013) and (2015)4026).

Author Contributions: Changwu Yue, Yuhong Lü, and Daishun Liu designed the study, prepared the manuscript, and wrote the paper; Changwu Yue, Yuhong Lü, and Miao Wang performed the biological experiments; Meiyun Shao, Yinyin Wang, Chengmin Deng, and Xiaoqian Li performed chemistry experiments; Shengyan Qian, Minghao Liu, and Ning Liu preformed the data analyses; Yinyin Wang, Yonglin $\mathrm{Hu}$, and Zehui Chen performed the antibacterial activity assay; Changwu Yue, Yuhong Lü, Yuxin Bao, and Ying Huang were the principal investigators of the project and provided the research funding.

Conflicts of Interest: The authors declare no conflict of interest.

\section{References}

1. Gribble, G.W. Biological activity of recently discovered halogenated marine natural products. Mar. Drugs 2015, 13, 4044-4136. [CrossRef] [PubMed]

2. $\mathrm{Xu}, \mathrm{G}$.; Wang, B.G. Independent evolution of six families of halogenating Enzymes. PLoS ONE 2016, 11, e0154619. [CrossRef] [PubMed]

3. Liao, L.; Chen, R.; Jiang, M.; Tian, X.; Liu, H.; Yu, Y.; Fan, C.; Chen, B. Bioprospecting potential of halogenases from Arctic marine actinomycetes. BMC Microbiol. 2016, 16, 34. [CrossRef] [PubMed]

4. Purrello, S.M.; Garau, J.; Giamarellos, E.; Mazzei, T.; Pea, F.; Soriano, A.; Stefan, I.S. Methicillin-resistant Staphylococcus aureus infections: A review of the currently available treatment options. J. Glob. Antimicrob. Resist. 2016, 7, 178-186. [CrossRef] [PubMed]

5. Kurosu, M.; Siricilla, S.; Mitachi, K. Advances in MRSA drug discovery: Where are we and where do we need to be? Expert Opin. Drug Discov. 2013, 8, 1095-10116. [CrossRef] [PubMed] 
6. Hoang, J.; Dersch-Mills, D.; Bresee, L.; Kraft, T.; Vanderkooi, O.G. Achieving therapeutic vancomycin levels in pediatric patients. Can. J. Hosp. Pharm. 2014, 67, 416-422. [CrossRef] [PubMed]

7. Reardon, J.; Lau, T.T.; Ensom, M.H. Vancomycin loading doses: A systematic review. Ann. Pharmacother. 2015, 49, 557-565. [CrossRef] [PubMed]

8. Dolgin, E. 'Game changer' antibiotic and others in works for superbug. Nat. Med. 2011, 17, 10. [CrossRef] [PubMed]

9. Gomez-Escribano, J.P.; Alt, S.; Bibb, M.J. Next generation sequencing of actinobacteria for the discovery of novel natural products. Mar. Drugs 2016, 14, 78. [CrossRef] [PubMed]

10. Yue, C.; Niu, J.; Liu, N.; Lü, Y.; Liu, M.; Li, Y. Cloning and identification of the lobophorin biosynthetic gene cluster from marine Streptomyces olivaceus strain FXJ7.023. Pak. J. Pharm. Sci. 2016, 29 (Suppl. 1), 287-293. [PubMed]

11. Walker, M.C.; Chang, M.C. Natural and engineered biosynthesis of fluorinated natural products. Chem. Soc. Rev. 2014, 43, 6527-6536. [CrossRef]

12. Kojiri, K.; Nakajima, S.; Fuse, A.; Suzuki, H.; Suda, H. BE-24566B, a new antibiotic produced by Streptomyces violaceusniger. J. Antibiot. (Tokyo) 1995, 48, 1506-1508. [CrossRef] [PubMed]

13. Lü, Y.; Yue, C.; Shao, M.; Qian, S.; Liu, N.; Bao, Y.; Wang, M.; Liu, M.; Li, X.; Wang, Y.; et al. Molecular genetic characterization of an anthrabenzoxocinones gene cluster in Streptomyces sp. FJS31-2 for the biosynthesis of BE-24566B and zunyimycin A. Molecules 2016, 21, 711. [CrossRef] [PubMed]

14. Chung, W.J.; Vanderwal, C.D. Stereoselective halogenation in natural product synthesis. Angew. Chem. Int. Ed. Engl. 2016, 55, 4396-4434. [CrossRef] [PubMed]

15. Kodali, S.; Galgoci, A.L.; Young, K.; Painter, R.; Silver, L.L.; Herath, K.B.; Singh, S.B.; Cully, D.; Barrett, J.F.; Schmatz, D.; et al. Determination of selectivity and efficacy of fatty acid synthesis inhibitors. J. Biol. Chem. 2005, 80, 1669-1677. [CrossRef] [PubMed]

16. Van Pée, K.H.; Milbredt, D.; Patallo, E.P.; Weichold, V.; Gajewi, M. Application and modification of flavin-dependent halogenases. Methods Enzymol. 2016, 575, 65-92. [PubMed]

17. Herath, K.B.; Jayasuriya, H.; Guan, Z.; Schulman, M.; Ruby, C.; Sharma, N.; MacNaul, K.; Menke, J.G.; Kodali, S.; Galgoci, A.; et al. Anthrabenzoxocinones from Streptomyces sp.as liver X receptor ligands and antibacterial agents. J. Nat. Prod. 2005, 68, 1437-1440. [PubMed]

18. Senn, H.M. Insights into enzymatic halogenation from computational studies. Front. Chem. 2014, 2, 98. [CrossRef] [PubMed]

19. Tian, J.; Chen, H.; Guo, Z.; Liu, N.; Li, J.; Huang, Y.; Xiang, W.; Chen, Y. Discovery of pentangular polyphenols hexaricins A-C from marine Streptosporangium sp. CGMCC 4.7309 by genome mining. Appl. Microbiol. Biotechnol. 2016, 100, 4189-4199. [CrossRef] [PubMed]

20. Clinical and Laboratory Standards Institute. Methods for Dilution Antimicrobial Susceptibility Tests for Bacteria that Grow Aerobically; Approved Standard M7-A7; Clinical and Laboratory Standards Institute: Wayne, PA, USA, 2012.

Sample Availability: Samples of the compounds zunyimycins A, B and C are available from the authors.

(C) 2017 by the authors; licensee MDPI, Basel, Switzerland. This article is an open access article distributed under the terms and conditions of the Creative Commons Attribution (CC BY) license (http:/ / creativecommons.org/licenses/by/4.0/). 